University of Nebraska - Lincoln

DigitalCommons@University of Nebraska - Lincoln

1987

\title{
Applications of the Semiclassical Spectral Method to Nuclear, Atomic, Molecular, and Polymeric Dynamics
}

M. L. Koszykowski

Sandia National Laboratories

G. A. Pfeffer

University of Nebraska at Omaha

D. W. Noid

Oak Ridge National Laboratory

Follow this and additional works at: https://digitalcommons.unl.edu/usdoepub

Part of the Bioresource and Agricultural Engineering Commons

Koszykowski, M. L.; Pfeffer, G. A.; and Noid, D. W., "Applications of the Semiclassical Spectral Method to Nuclear, Atomic, Molecular, and Polymeric Dynamics" (1987). US Department of Energy Publications. 37. https://digitalcommons.unl.edu/usdoepub/37

This Article is brought to you for free and open access by the U.S. Department of Energy at DigitalCommons@University of Nebraska - Lincoln. It has been accepted for inclusion in US Department of Energy Publications by an authorized administrator of DigitalCommons@University of Nebraska - Lincoln. 


\title{
Applications of the Semiclassical Spectral Method to Nuclear, Atomic, Molecular, and Polymeric Dynamics ${ }^{a}$
}

\author{
M. L. KOSZYKOWSKI, ${ }^{b}$ G. A. PFEFFER, ${ }^{c}$ \\ AND D. W. NOID ${ }^{d}$ \\ ${ }^{b}$ Combustion Research Facility \\ Sandia National Laboratories \\ Livermore, California 94550 \\ ${ }^{c}$ Department of Chemistry \\ University of Nebraska at Omaha \\ Omaha, Nebraska 68182-0109 \\ ${ }^{d}$ Chemistry Division \\ Oak Ridge National Laboratory \\ Oak Ridge, Tennessee 37831 \\ and \\ Department of Chemistry \\ University of Tennessee \\ Knoxville, Tennessee 37996-1600
}

\section{INTRODUCTION}

Nonlinear dynamics plays a dominant role in a variety of important problems in chemical physics. Examples are unimolecular reactions, ${ }^{1}$ infrared multiphoton decomposition of molecules, ${ }^{2}$ the pumping process of the gamma ray laser, ${ }^{3}$ dissociation of vibrationally excited state-selected van der Waals's complexes, ${ }^{4}$ and many other chemical and atomic processes. The present article discusses recent theoretical studies on the quasi-periodic and chaotic dynamical aspects of vibrational-rotational states of atomic, nuclear, and molecular systems using the semiclassical spectral method (SSM). ${ }^{5}$ We note that the coordinates, momenta, and so on, are found using classical mechanics in the studies included in this review. Consequently, certain processes of quantum mechanical origin, such as tunneling, have not been investigated in this framework. However, the projects to be described, undertaken using classical or semiclassical techniques, yield excellent results and explain many aspects of the underlying dynamics.

\footnotetext{
${ }^{a}$ This submitted manuscript has been authored by a contractor of the U.S. Government under Contract No. DE-AC05-84OR21400. Accordingly, the U.S. Government retains a nonexclusive, royalty-free license to publish or reproduce the published form of this contribution, or allow others to do so, for U.S. Government purposes. M. L. Koszykowski's work was supported by the U.S. Department of Energy. D. W. Noid's research was sponsored by the Division of Materials Sciences, Office of Basic Energy Sciences, U.S. Department of Energy, under Contract No. DE-AC05-84OR21400 with Martin Marietta Energy Systems, Inc.
} 
The SSM technique can be applied to any autocorrelation function [e.g., of coordinates $x(t)$ or $y(t)$, momenta $\mathrm{p}_{x}(t)$ ] or any dynamical variable [e.g., the dipole moment $\mu(t)$ ]. Below, we shall use $\chi(t)$ to denote any quantities that couple to a perturbation that causes a transition.

The infrared absorption-band shape function $I(\omega)$ [or in the case of any dynamical variable, $\chi(t)$, the power spectrum or spectral density] is related to the Fourier transform of its autocorrelation function $C(t)$ in the well-known way:

$$
I(\omega)=\frac{1}{2 \pi} \int_{-\infty}^{+\infty} C(t) \exp (-i \omega t) d t
$$

where

$$
C(t)=\langle\chi(0) \chi(t)\rangle \text {. }
$$

The average ( ) indicates an average over an ensemble appropriate to the problem (this ensemble will be described later). $C(t)$ has the stationary property

$$
\langle\chi(0) \chi(t)\rangle=\langle\chi(\tau) \chi(t+\tau)\rangle,
$$

and because $\chi(t)$ is real, it also has the property of being an even function of $t$. For classical trajectories, equation 1.1 then reduces to

$$
I(\omega)=\frac{1}{2 \pi} \lim _{T \rightarrow \infty} \frac{1}{2 T}\left\langle\left|\int_{0}^{2 T} \chi(t) \exp (-i \omega t) d t\right|^{2}\right\rangle
$$

in both the quasi-periodic and chaotic regimes described in the next paragraph. Equation 1.4 indicates that we can evaluate the spectra from a single classical trajectory generated from a time-independent Hamiltonian of the system studied. The choice of this trajectory will be discussed later.

The method has been most extensively used to characterize intramolecular dynamics. The understanding of nonlinear dynamical systems received tremendous attention during the late 1970 s and the 1980 s. During this time, it was found that a

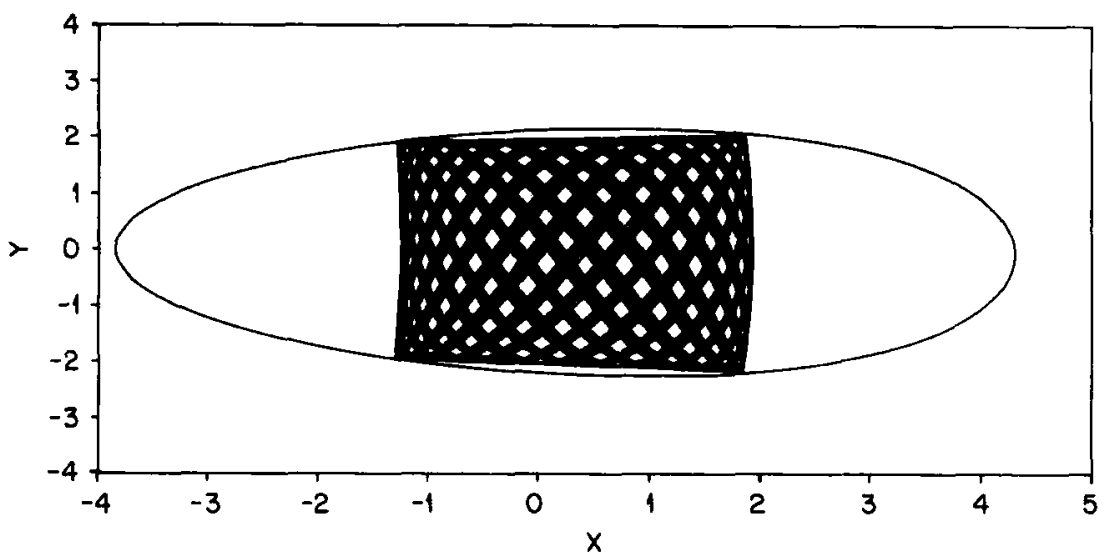

FIGURE 1. A typical boxlike quasi-periodic trajectory for a two-mode system. 


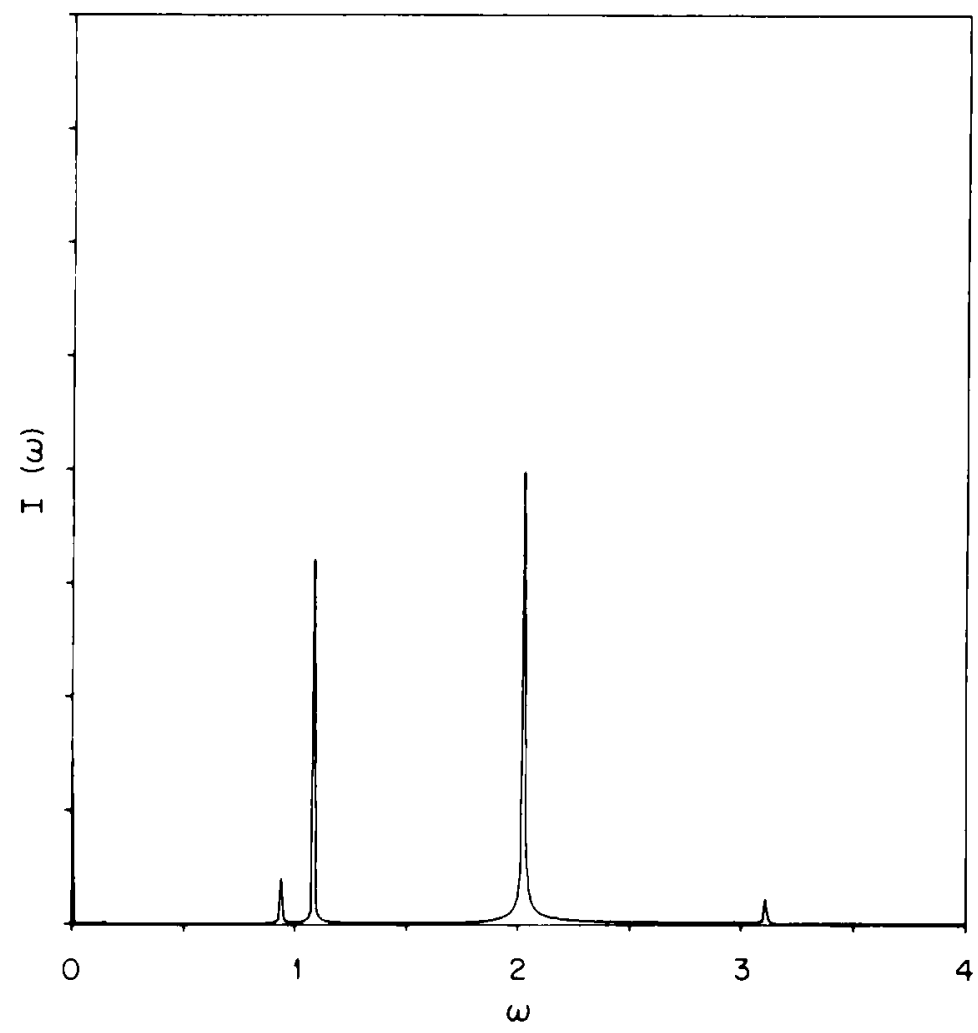

FIGURE 2. Spectra of the $x$ and $y$ coordinates of the trajectory shown in FIGURE 1 .

dynamical system could exhibit two very different types of motion. A stable, more familiar type, termed quasi-periodic, is found for many ground-state systems. After a certain amount of excitation in the system, the motion becomes "random" and is termed chaotic. A recent review of molecular chaos by Noid, Koszykowski, and Marcus (NKM) can be found in reference 6. In the quasi-periodic regime, the motion can be decomposed into a Fourier series with a few fundamental frequencies and overtones. A typical quasi-periodic trajectory for a two-mode system is shown in FIGURE 1. The spectrum of the coordinates, say $x+y$, is shown in FigURE 2 and, as expected, consists of a small number of sharp lines. In the chaotic regime, the trajectories sample much more (if not all) of the allowed phase-space, as shown in Figure 3. The spectrum, as expected, becomes broad and is shown in Figure 4. The use of correlation functions and spectra as tools for examining chaotic behavior has been extensively discussed in reference 6 .

A number of possible choices have been explored for initial conditions of the trajectory used to evaluate equation 1.4. The methods are based upon quantized action integrals. Many years ago, Einstein ${ }^{7}$ proposed a quantization of systems not permitting separation of variables: One finds canonical invariants, namely, the action variables $J_{i}$, 


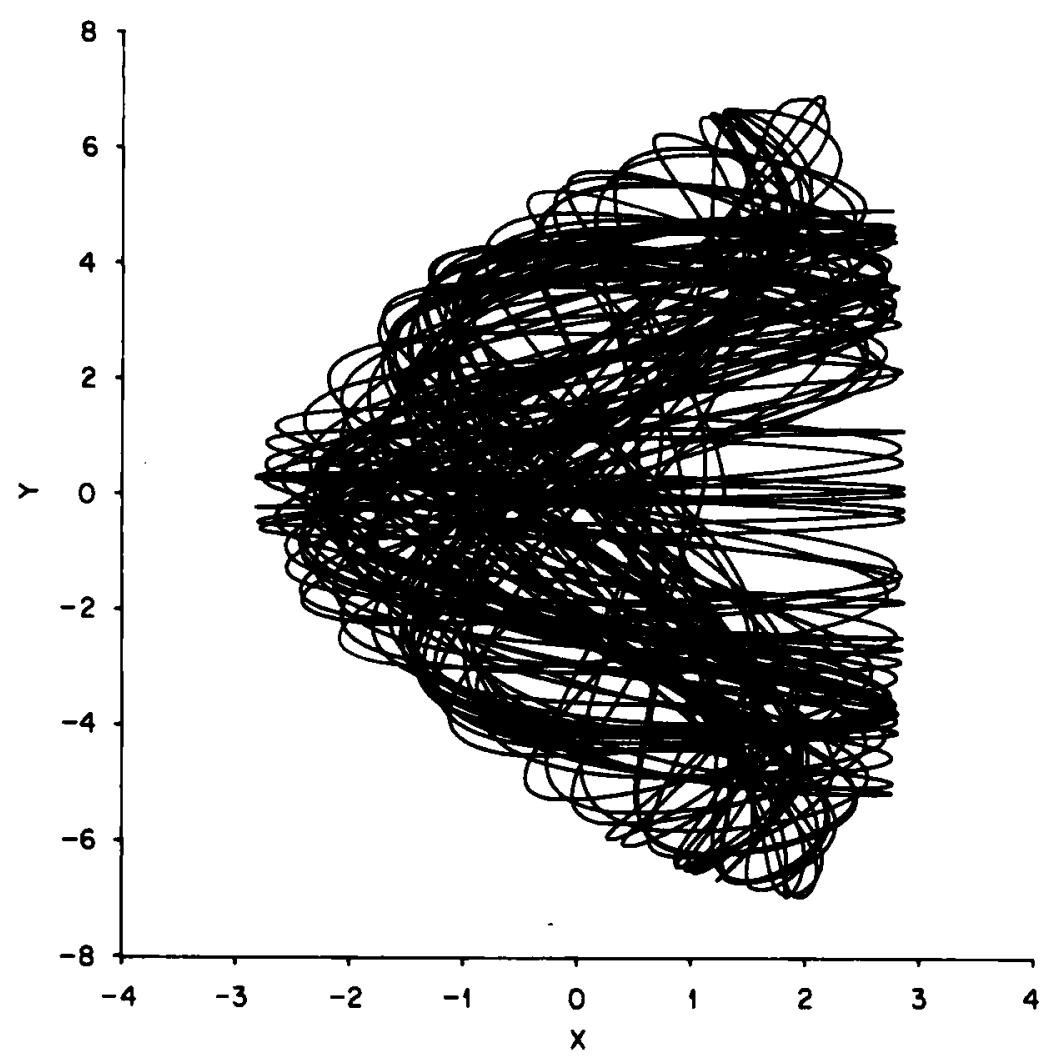

FIGURE 3. A chaotic trajectory for a two-mode system.

and quantizes them

$$
J_{i}=\oint \tilde{\mathrm{p}} \cdot d \tilde{\mathrm{q}}-n_{l} h,
$$

where the different $J_{i}$ 's are obtained by integrating over topologically independent paths; $\bar{q}, \tilde{p}$ denote canonically conjugate coordinates and momenta, respectively. The theory was further developed by $\mathrm{Keller}^{8}{ }^{8}$ who showed how fractional terms arose:

$$
J_{i}=\oint \tilde{\mathrm{p}} \cdot d \tilde{\mathrm{q}}=\left(n_{i}+\delta_{i}\right) h,
$$

where $\delta_{i}$ is a known constant, usually being 0 or $1 / 2$, depending on the degree of freedom. He further showed how to evaluate these integrals for a number of nonseparable systems that had zero potential energy within a confined region and that rose to infinity on the boundary of that region.

The SSM technique has been greatly extended in the past ten years, and now routine evaluation of equation 1.4 for multidimensional systems including resonances is possible. Originally, trajectories with $n_{1}=0,1, \ldots$ were used as initial conditions for the spectra from level $n$. Later, it was shown for the Morse oscillator that the use of 
$\bar{n}=(n+m) / 2$ for $n \rightarrow m$ transitions was preferable. ${ }^{9}$ For other potential functions, slightly more accuracy can be obtained using other correspondence principles.

\section{APPLICATION TO ATOMIC/MOLECULAR SYSTEMS}

\section{Polyatomic Molecules}

The spectral analysis method has been applied to a large number of generic molecular Hamiltonians - the intent usually being to either develop or test new semiclassical techniques, or, more frequently, as a tool for studying the nonlinear dynamics and ramifications of chaos. A much smaller set of work has focused on Hamiltonians intended to represent a specific molecular or atomic system. In this section, we will discuss examples of both types of problems with an analysis on the work related most closely to molecular systems.

The choice of initial conditions for the trajectories is crucial to the success of this method. In their original work, $\mathrm{NKM}^{5}$ used a primitive average of frequencies from zeroth order eigentrajectories. While this choice produced encouraging results, it was not until Koszykowski et al. ${ }^{9}$ proposed that the $\bar{n}=\left(n_{i}+n_{f}\right) / 2$ trajectory be used that a firm semiclassical basis for the initial condition was established. They applied this analytically to the Morse oscillator and demonstrated exact agreement with quantum calculations. In this work, it was also demonstrated, for the first time, that the spectral

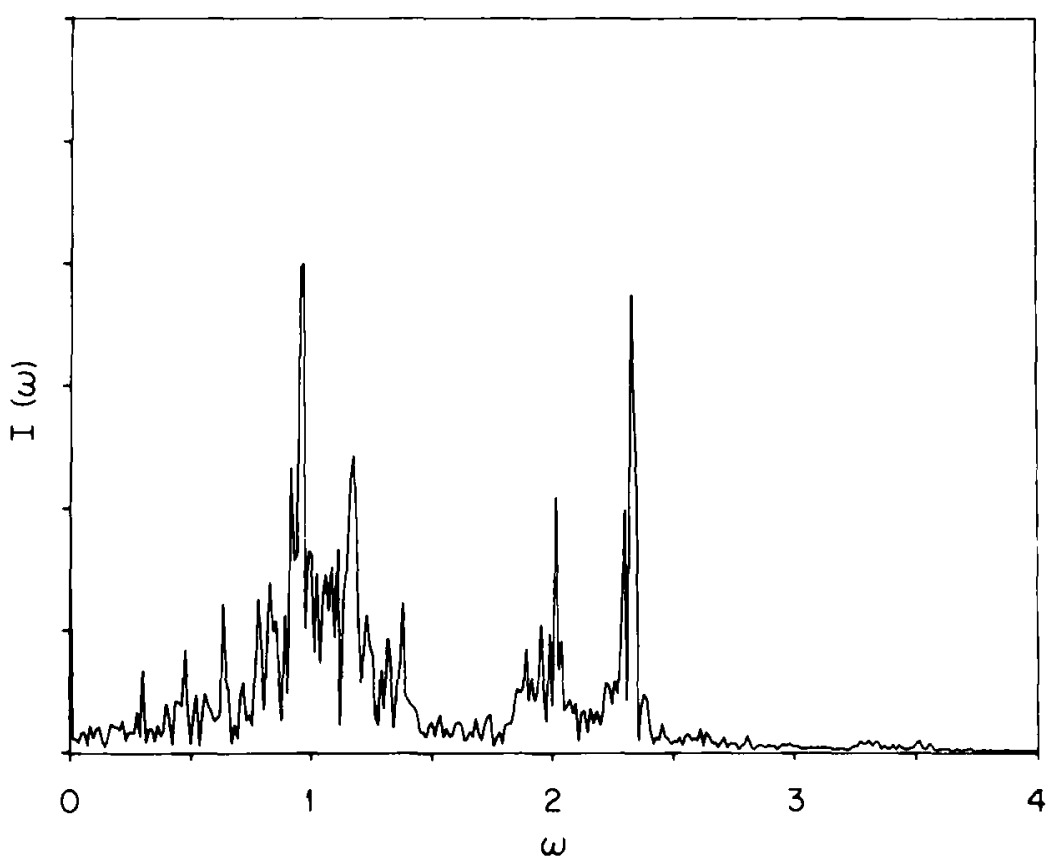

FIGURE 4. Spectra of the $x$ and $y$ coordinates of the trajectory shown in FIGURE 3. 
analysis method could be used on a realistic surface where they calculated frequencies and intensities for OCS. ${ }^{9}$ These workers also demonstrated that frequencies and intensities of overtones and combination bands could be easily obtained, even when they were many orders of magnitude weaker than the fundamental. ${ }^{9}$

Wardlaw et $a l .{ }^{10}$ have quantitatively tested the SSM technique on a wide variety of two-mode systems with several types of resonance interaction. The results were in very good agreement with exact quantum results and were considerably better than results obtained by low order perturbation theory. Later, Herbst and Noid" showed that even accurate bound-free and free-free transitions could be obtained for quasi-periodicquasi-bound states. ${ }^{12}$

In another study, Liu, Noid, and Koszykowski ${ }^{13}$ performed a spectral analysis of the vibrational motion of a realistic model of water. As the energy of the molecule increases beyond a certain transition value, the spectra changes from consisting of well-resolved lines to a much broadened one, indicating chaotic motion. Isotopic substitution that lowers the symmetry of the molecule reduced the transition energy because additional anharmonicities are introduced. Freezing the bending motion has a drastic effect on the motion of the molecules. Finally, a cross-correlation function was defined whose spectrum exhibits the same features as that of the self-correlation functions.

Stine and Noid ${ }^{14}$ employed the spectral analysis method as a basis for studying diatomic molecules using numerically accurate potential functions. These potential functions were developed using the Rydberg-Klein-Rees (RKR) method for analyzing experimental spectral data. They calculated dipole matrix elements for $\mathrm{HF}, \mathrm{CO}$, and NO. They demonstrated that if the dipole function has a maximum at an intermolecular separation larger than the equilibrium configuration, the intensity of $\Delta \boldsymbol{n}=2$ transitions will be greater than $\Delta \boldsymbol{n}=1$ transitions for highly excited states. This is in complete contradiction to conventional wisdom based upon a harmonic oscillator model. They also presented an inversion method ${ }^{15}$ where experimental results could be used to predict the dipole moment function. The method was applied to HF with excellent results.

A number of other gas-phase studies have applied the spectral analysis method to molecular problems. Hansel ${ }^{16}$ related the width of broadened chaotic spectra to a value of the $K$ entropy (see reference 6 ). He also discussed these concepts using a model of ozone. Smith et al. ${ }^{17}$ also used the SSM technique on ozone and found the frequencies to be more accurate than an SSCF calculation. The semiclassical self-consistent field (SSCF) method was proposed a few years ago by Ratner and Gerber. ${ }^{18}$ It reduces an $n$-coupled oscillator potential to $n$ single-mode potentials that are determined selfconsistently. These are then quantized semiclassically using the usual Bohr-Sommerfeld quantization rule. Later, Poppe ${ }^{19}$ studied rotational spectra of $\mathrm{SF}_{6}$ and also related them to the maximal Lyapunov exponent. Noid and Stine ${ }^{20}$ and Martin and Wyatt ${ }^{21}$ have all used this method in studies of IR multiphoton processes.

General studies of chaos using this method include Powell and Percival,22 who computed a spectral entropy using the SSM technique. Farantos and Murrell, ${ }^{23}$ Klemperer and co-workers, ${ }^{24}$ Swamy and Hase, ${ }^{25}$ Brickmann et al. ${ }^{26}$ Lopez et al., ${ }^{27}$ and Demontis et al. ${ }^{28}$ all studied chaos in various molecular energy transfer processes. The study of chaos in molecular systems has been a much discussed topic, and the spectral analysis method proves to be a useful tool in characterizing different regions of phase-space. 
In another study of vibrational spectroscopy, Koszykowski et al. ${ }^{29}$ derived an expression for line shapes valid in both the quasi-periodic and chaotic regimens and for densities ranging from ultrahigh vacuum to several atmospheres. The Laplace transform of the dipole correlation function is given by

$$
C(s)=C_{0}\left(s+\frac{i}{\tau}\right) /\left[1-\left(\frac{1}{\tau}\right) C_{0}\left(s+\frac{i}{\theta}\right)\right],
$$

where $C_{0}$ is the zero pressure limit and $\tau$ is a relaxation rate due to collisions (calculated in the binary collision approximation). They found, as is well known, that for increasing collisional relaxation rates, the spectrum becomes broadened, shifted, overlapped, and eventually merged. A sequence of spectra for increasing collisional rates is shown in FIGURE 5 and clearly demonstrates this effect. Likewise, as the chaotic nature of the molecule increases, a similar effect is observed and the individual spectral lines merge more quickly. This can be clearly observed in FIGURE 6, as contrasted to FIGURE 5, where it is clear that chaos also affects the wings of the lines. Careful experiments at various pressures may be capable of distinguishing this effect from other complex variables in the line shape. Such a study would be an interesting way to observe chaos in the presence of collisions, along with being a generalization of the quantum beat experiments being done in a number of laboratories.

Koszykowski et $a l^{30}$ and Noid et al..$^{31,32}$ have also used correlation functions and spectra in new ways to extract fundamental information about the dynamics of molecular vibrations. In reference 30 , they demonstrated that microcanonically averaged correlation functions decay from some initial value to that predicted by a microcanonical average over phase-space of the variables. Indeed, for these nonlinear systems, phase-space and time averages are interchangeable and the Rice-Ramsperger-Kassel-Marcus (RRKM) theory's basic assumption is valid.' RRKM is a theoretical treatment of unimolecular reactions. The first calculations of semiclassical intensities using primitive initial conditions are presented in reference 31 , along with a derivation of intensities for the SSM technique. It was also demonstrated in this work that at a given total energy, one could locate chaotic spectra that are quite different. This led to the postulate that microcanonically averaged semiclassical spectra should correspond to quantum spectra generated from a wave packet composed of energies within a distance $\Delta E$ of the semiclassical state. Noid et al. ${ }^{33}$ also have shown that the spectra of weak combination lines could be used to obtain the splitting between states degenerate in the primitive semiclassical method. In addition, by using correlation functions from classical trajectories, Muckerman et al. ${ }^{34}$ demonstrated a method to determine the local-mode versus normal-mode character of molecular vibrations in a two-mode model of $\mathrm{H}_{2} \mathrm{O}$.

Very recently, we have introduced a method ${ }^{33}$ for obtaining classical trajectories directly from a grid of points representing a potential energy function. Hamilton's equations were obtained by using an automated routine to generate multidimensional splines under tension through the total energy points. The output includes the derivatives at any arbitrary location in coordinate space that were used to numerically generate Hamilton's equations. The trajectories obtained with this method were indistinguishable from those generated with an analytic potential function and with analytic derivatives. This technique can now be used to couple directly with an electronic structure code to generate completely ab initio trajectories and spectra. No 

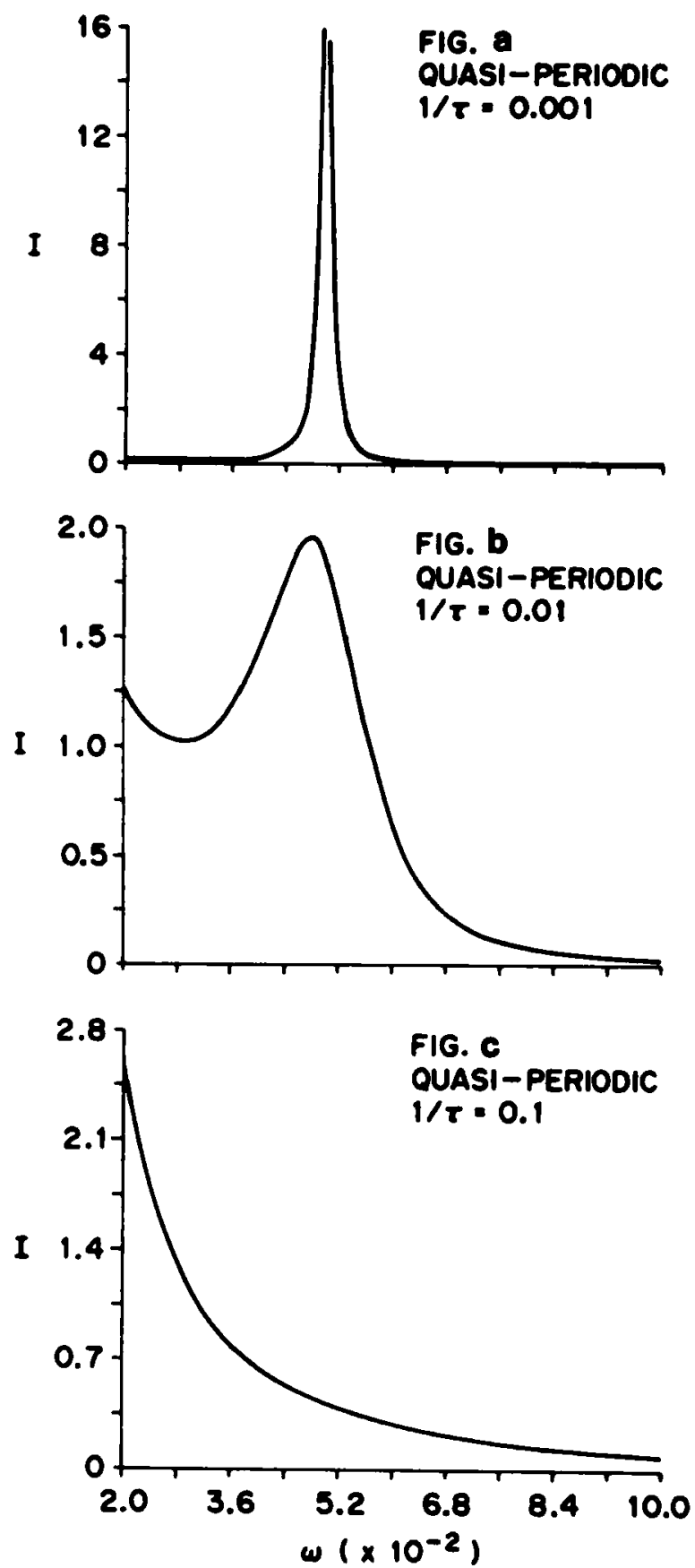

FIGURE 5. The dependence of the $\omega=0.5$ peak for the Hénon-Heiles system with various values of collisional relaxation in the quasi-periodic regime. 

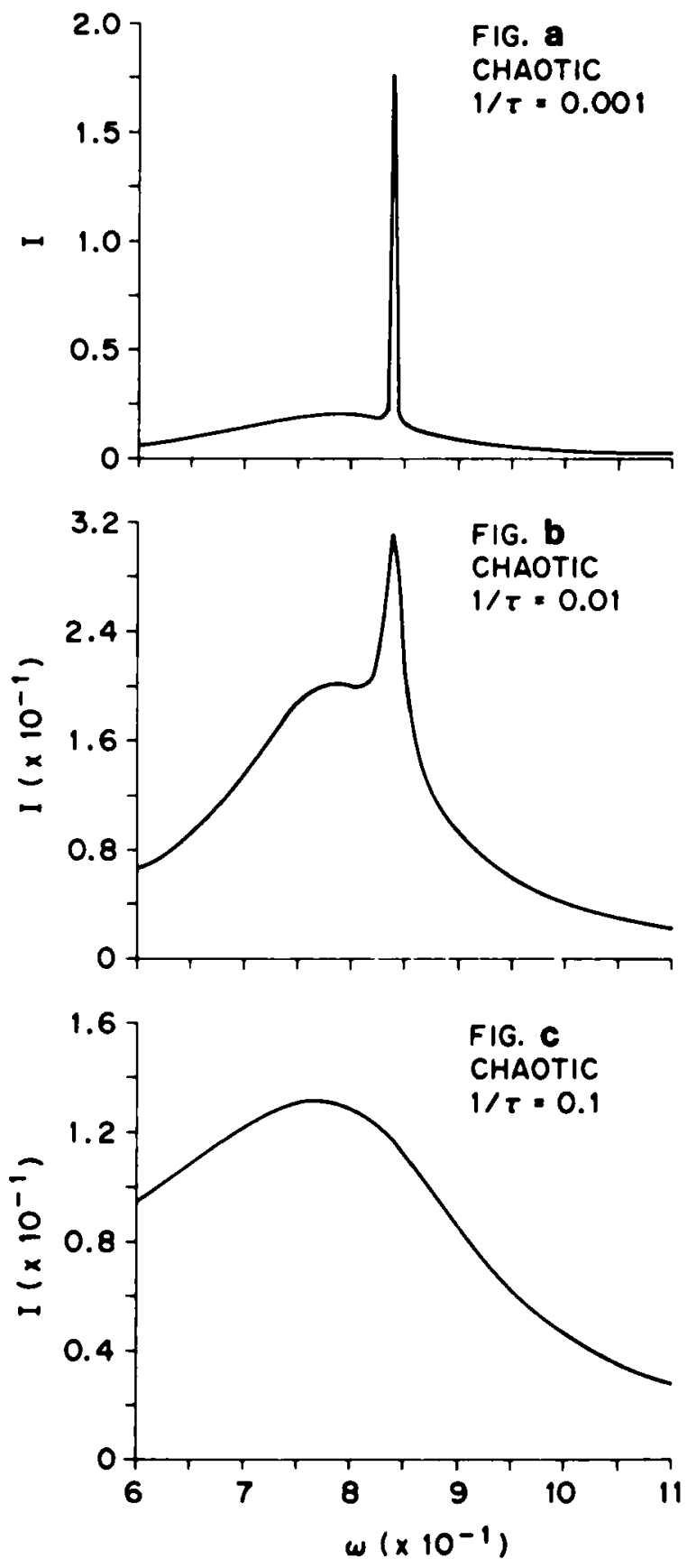

FIGURE 6. Same as FIGURE 5, but for chaotic motion. 
analytic functions are imposed on the points and no errors are introduced by a fitting procedure. Thus, we can examine dynamics problems without the ambiguity of knowing a good representation of the potential function or what the best near separable coordinate system may look like, if there is one.

As a demonstration problem, we used fourth order many-body perturbation theory and a 6-31G* basis set ${ }^{35}$ to calculate a grid of total energy points for ozone. This grid was combined with the spline under tension code to generate trajectories and spectra for ozone. A spectra for the ground state is shown in Figure 7. It is interesting to note that a harmonic analysis of the energy points predicts frequencies that are at least 100 $\mathrm{cm}^{-1}$ further from the experimental values than those in FIGURE 7. Analytic surfaces

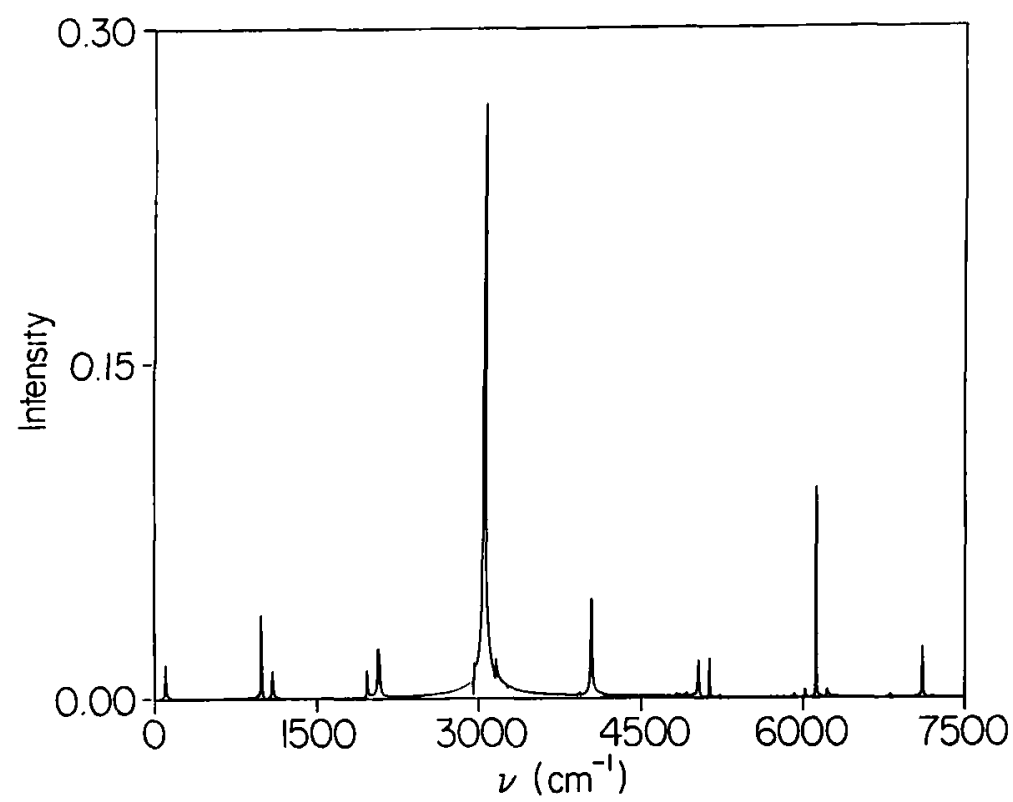

FIGURE 7. Spectra of a two-mode model of $\mathrm{O}_{3}$ using a numerical grid of potential energy values and dipole moments for the ground state.

for ozone have shown significant regions of chaotic motion. In our study, we found no chaos at $E=15,000 \mathrm{~cm}^{-1}$, which is the highest energy studied. Koszykowski et al. ${ }^{36}$ applied the spectral method to examine the EELS spectra of ice multilayers on Ru. They used a polynomial potential fit to ab initio calculations of the local O-H mode energetics. The $\mathrm{OH}$ stretch frequency of hydrogen pointing into and out of the multilayer agreed with experiment to within $25 \mathrm{~cm}^{-1}$. However, edge hydrogens in the calculation produced frequencies where there were clearly no experimental lines present. One possibility for the discrepancy is the limited potential used because electron structure calculations on metals are unreliable except in reproducing trends. Tully et $a l^{37}$ have recently extended the method to include a stochastic procedure aimed at vibrational relaxation. They performed a totally $a b$ initio line-shape 
calculation for $\mathrm{H}$ on $\mathrm{Si}$ surfaces in excellent agreement with experiment. Gadzuk ${ }^{38}$ and Adams $^{39}$ have both recently reported good results with the SSM technique for molecules absorbed on surfaces. Adams studied the vibrational dynamics of $\mathrm{HCl}$ on an Ar surface and calculated both the ro-vibrational and vibrational spectra. These later calculations were very successful in studying vibrational spectra on insular and semiconductor surfaces. A successful ab initio application to conductor surfaces must await further breakthroughs in the quality of electronic structure calculations on these systems.

\section{Atomic and Nuclear Systems}

The present method has also proven useful for calculation of atomic and nuclear spectra. Transition frequencies and intensities for excited states of atomic hydrogen in a strong magnetic field were calculated by the SSM technique. ${ }^{40}$ The states considered had a principal quantum number of 30 and $L_{z}=1$ for fields of up to 7 tesla. The spectra for states there that are labeled as librators and rotators are found to be qualitatively different, especially for the spectral component perpendicular to the field. In addition to the zero-field Kepler line, a new intramanifold transition was found at low frequency. The frequencies and intensities were found to be a sensitive function of the field strength and of the particular state of the Coulomb manifold involved.

A field-induced transition at low frequency was also observed, which is in agreement with predictions from perturbation theory; this agreement supports the accuracy of the computed intensities. The frequency and intensity are strongly dependent upon both the field strength and on the quantum state in the manifold. Qualitatively correct behavior is found for the intermanifold transition, but the accuracy of the calculation is not sufficient to identify the upper state uniquely. This method also found polarized spectra parallel and perpendicular to the field to be qualitatively different. In part, this effect is due, in quantum terms, to the $z$ - and $x$-direction spectra obeying different selection rules. However, in addition, the distinction reflects the different natures of the orbits for the two classes of states mentioned above, which is demonstrated by the differences in the $x$-spectra. Spectral parameters in this transition regime would be difficult to obtain by other methods.

Finally, in this subsection, we now discuss a coupled nucleon electronic system. In order to model the energy transfer from an inner core electron to an excited nuclear proton, we have chosen to use the nuclear shell model approach for an independent valence nucleon. This model serves to introduce the important characteristicsprimarily energy level spacing and nuclear size effects-which are orders of magnitude disparate from the electronic characteristics. In this model, a valence excited proton is bound as an independent particle to the nuclear core using an effective Wood Saxons potential. ${ }^{41}$ The electron is bound to both the core of protons and the valence proton using the normal Coulomb potential. Our Hamiltonian is

$$
H=\frac{1}{2 m_{N}}\left(P_{N}^{2}+\frac{L_{N}^{2}}{R_{N}^{2}}\right)+V_{N}\left(R_{N}\right)+\frac{1}{2 m_{e}}\left(P_{e}^{2}+\frac{L_{e}^{2}}{r_{e}^{2}}\right)-\frac{Z}{r_{e}^{2}}+H_{\mathrm{INT}},
$$

where $m_{N}$ is the mass of a nucleon, $P_{N}$ is the nucleon momentum, $L_{N}$ is the nucleon 
orbital angular momentum, $R_{N}$ is the radial position of the nucleon, and

$$
V_{N}\left(R_{N}\right)=V_{0} /\left(1+e^{\left(R_{N}-R\right) / A}\right)
$$

is the nucleon potential. In this potential, $V_{0}$ is the well depth and $A$ is the surface diffusivity parameter. The quantities, $m_{e}, P_{e}, L_{e}$, and $r_{e}$, are the corresponding electron properties. The Coulomb potential is included for the interaction of the electron with the closed shell nuclear core and is given by

$$
H_{\mathrm{INT}}=-\frac{1}{r_{\text {ep }}}
$$

where $r_{e p}=\left|\tilde{r}_{e}-\tilde{R}_{N}\right|$.

In our study, we considered the coupling of the $1 S$ electronic state with the excited nucleon state for closed shell +1 nucleons. The number of protons considered are 29, 51, and 83. In Figure 8, we present the unpolarized spectra for the coupled electron-nucleon model, with the electronic state being $1 S$ and a $3 F$ proton state with $Z=83$. In FIGURE 9, we present the uncoupled spectrum for the same nuclear-electron state.

As can be observed from these figures, a large number of lines with spacing corresponding to electronic transition are observed. A smaller number of larger lines occur at about 1 and $3 \mathrm{MeV}$ (not shown) that correspond to pure nuclear transitions. Interestingly enough, the spectra in FIGURE 8 appear to have some features of chaos,

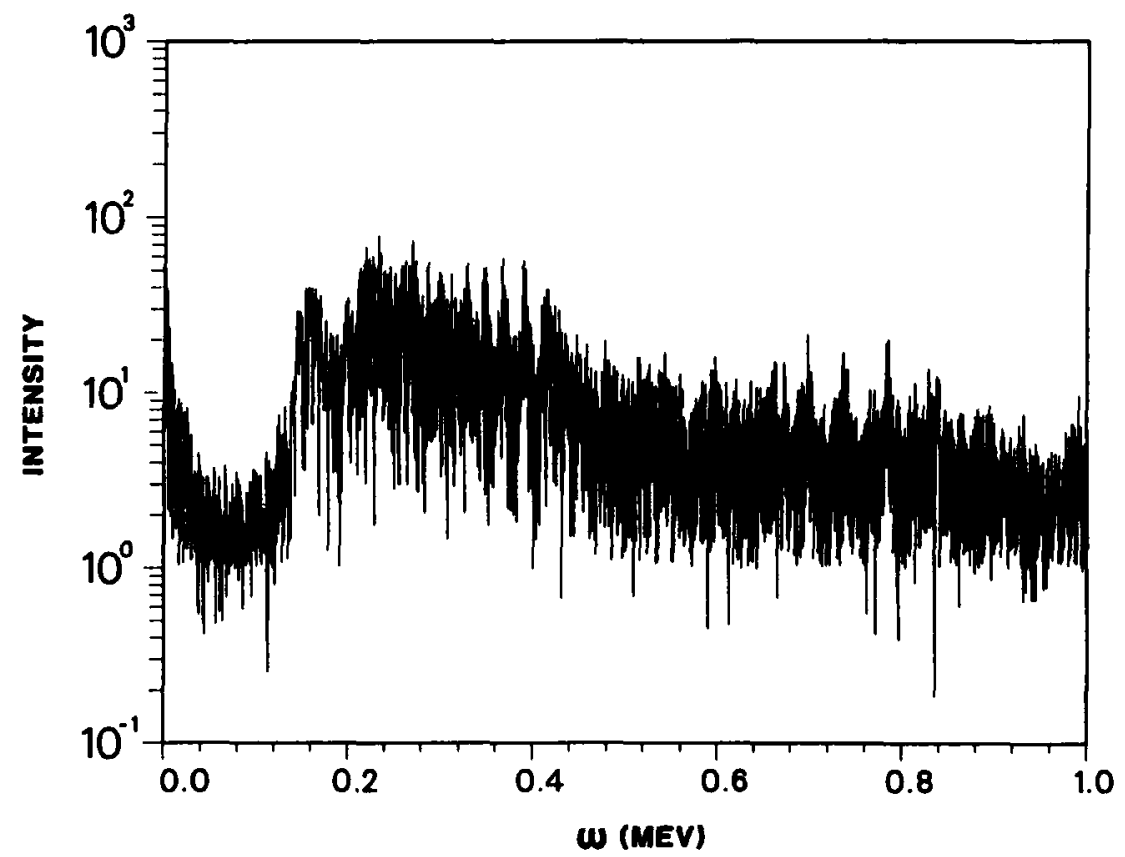

FIGURE 8. Unpolarized spectra for the nuclear-electron model showing chaotic motion. 


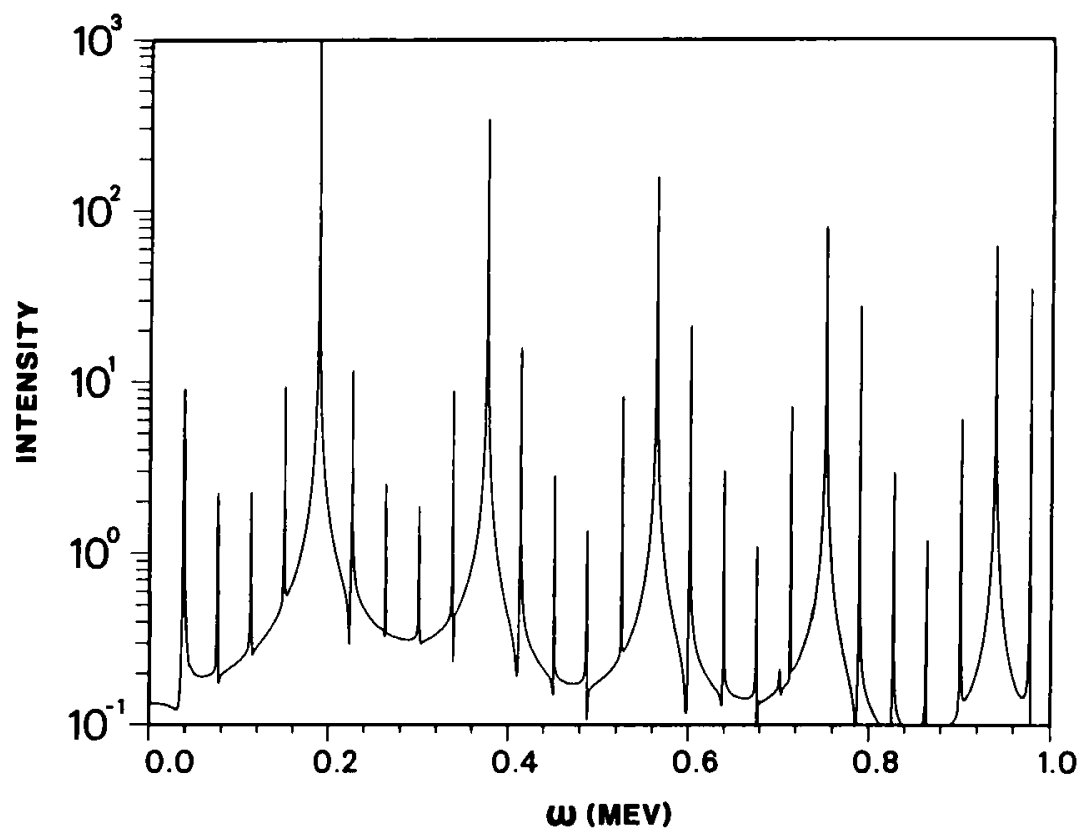

FIGURE 9. Same as FIGURE 8, but for the uncoupled case.

which indicates that the classical dynamics is strongly coupled. For the $Z=29$ case, none of the spectra appear to have chaotic features, and therefore the coupling is much weaker.

Among the preliminary conclusions of this study are: ${ }^{42}$

- high $Z$ produces the greatest distortion of the nuclear-electron spectra;

- use of the Langer correction ${ }^{43}$ for the $I S$ state is very important (because old quantum theory orbits and spectra failed to be chaotic);

- finally, the SSM technique is a very practical technique to study the very subtle coupling in this model.

\section{Polymeric Systems}

In this section, we present some preliminary results concerning the transition from quasi-periodic to chaotic motion in a long linear diatomic chain. A few studies have been reported for linear atomic chains, ${ }^{44}$ but even less work has been reported using polymeric diatomic chains. ${ }^{45}$ We also further extended the previous research by studying longer chain lengths: the longest chains considered before had contained $\sim 1000$ atoms; we have employed $20-3200$ atoms (10-1600 diatoms). The motivation for both our investigations and those preceding us has been twofold: to find an appropriate criteria to detect or predict the onset of chaos in systems with many modes, and to examine the stochastic threshold as a function of chain length, initial excitation, 
and other chain attributes. Such work is applicable to energy transfer in polymers and in solids.

Our model chain mimics a linear array of $A B$ molecules where the $A-B$ bond is a typical chemical bond and the $B \cdots A$ interaction is much weaker. A Morse oscillator is chosen for the $A B$ bond and has the form,

$$
V(r)=D\left(e^{-2 a(r-r e)}-2 e^{-\alpha(r-r e)}\right),
$$

where $r$ is the $A-B$ distance. The $B \cdots A$ interaction is given by a Lennard-Jones potential,

$$
V(R)=4 \epsilon\left[\left(\frac{\sigma}{R}\right)^{12}-\left(\frac{\sigma}{R}\right)^{6}\right],
$$

where $R$ is the distance between the molecular centers of mass. Our choice of parameters is appropriate for a chain of HF molecules. In the HF chain, the F ...H interaction is an example of hydrogen bonding and the center of mass of the molecule can be considered to be at the $F$ nucleus.

A one-dimensional "temperature" was calculated from the kinetic energy at each time step. This temperature remained reasonably constant after an initial equilibration period. The spectra were generated by a fast Fourier transform of the sum of all the particle coordinates at each time step. The initial $\mathrm{H}$ and $\mathrm{F}$ positions were first chosen so that each particle sat at the minimum distance of the Morse oscillator and the Lennard-Jones potential relative to that particle's nearest neighbor. A random displacement (either positive or negative) from that minimum position was then

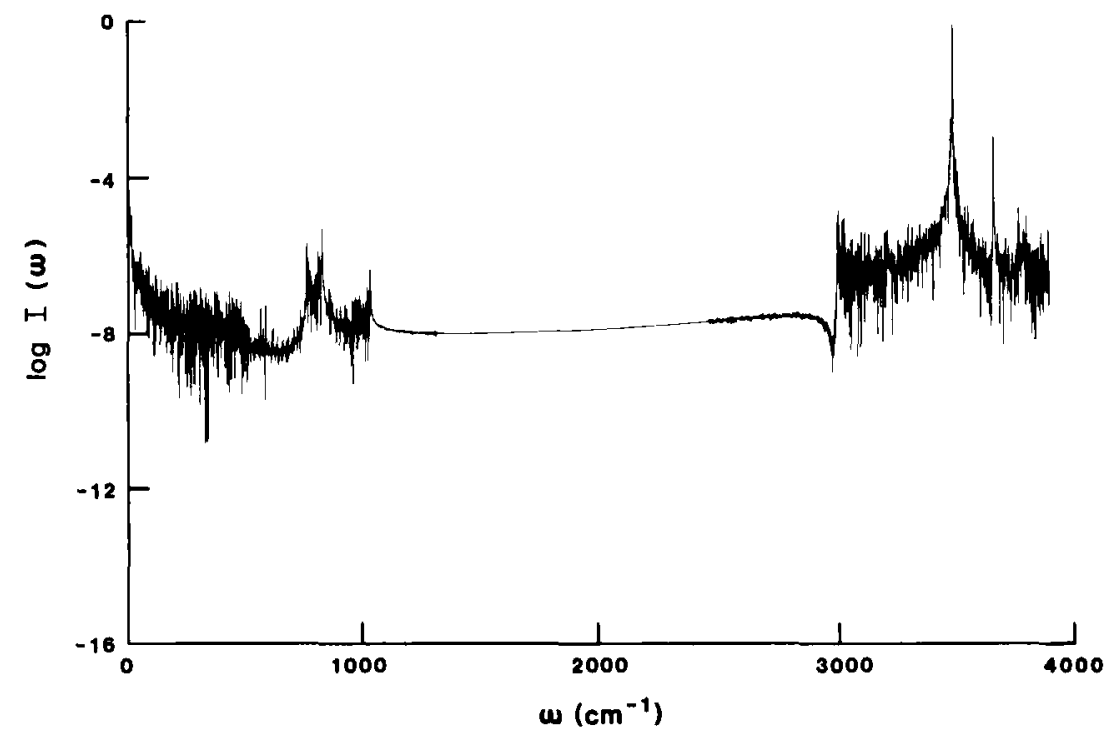

FIGURE 10. Spectra of a polymeric HF chain with 1280 atoms in the quasi-periodic regime for a low temperature $(1 \mathrm{~K})$. 


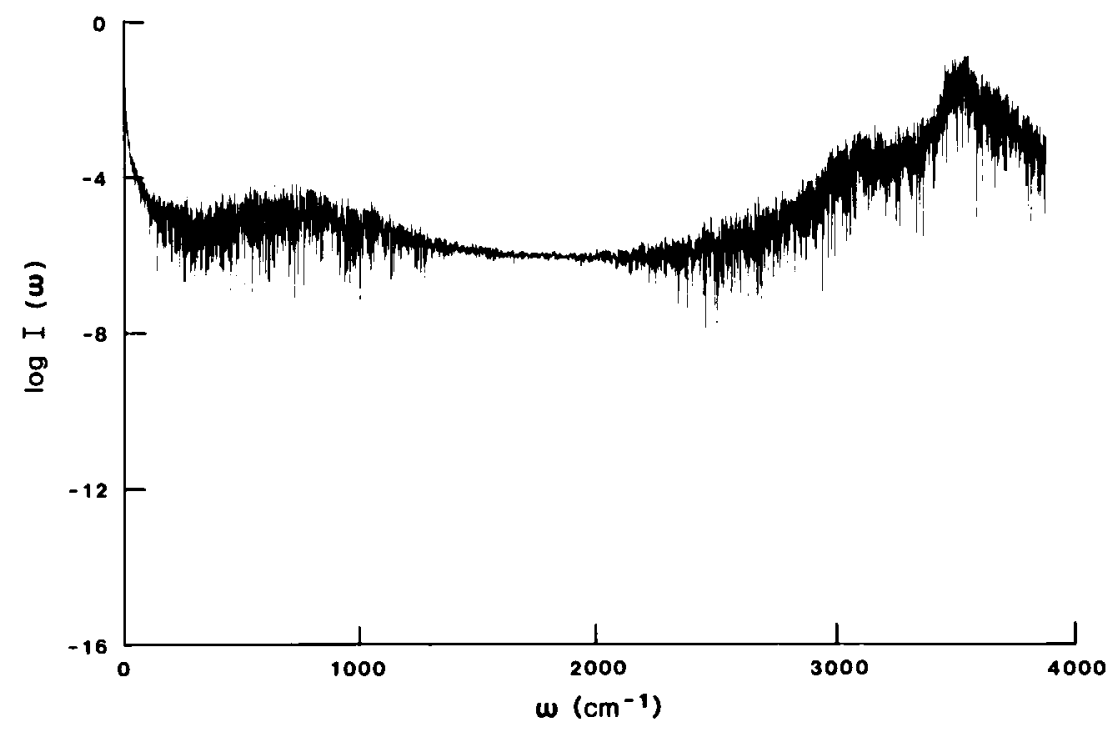

FIGURE 11. Same as FIGURE 10, but for a higher temperature $(135 \mathrm{~K})$ and chaotic motion.

introduced to give the final positions. By changing the maximum position displacement parameter and the maximum momentum parameter, the temperature could be changed. The ends of the chain were not fixed (in contrast to several previous studies).

The spectra clearly showed a low frequency set of peaks $\left(\$ 100 \mathrm{~cm}^{-1}\right)$ and a high frequency set of peaks $\left(\sim 3000 \mathrm{~cm}^{-1}\right)$. The choice of initial conditions described above gives, in the low temperature case, nearly $100 \%$ excitation of the high frequency modes. The conclusions reported here deal only with these initial conditions, and conclusions for other choices of initial conditions may be different.

FIGURE 10 shows the power spectrum for 1280 particles ( $640 \mathrm{HF}$ molecules) at a nominal temperature of $1 \mathrm{~K}$. The log of the intensity from the fast Fourier transform is actually displayed rather than the intensity itself because the low frequency peaks are more easily seen in the log plot. Well-separated sharp peaks can be seen in the range $0-1300 \mathrm{~cm}^{-1}$ and in the range $3250-3924 \mathrm{~cm}^{-1}$. There is an intermediate region where little noise and no peaks are seen. Based upon the appearance of the spectrum, the system appears to be quasi-periodic at this temperature. FIGURE 11 shows the (log) power spectrum for the same system at $135 \mathrm{~K}$, where no sharp peaks are seen and where the noise-free intermediate region has disappeared. Based upon the appearance of this spectrum, the system appears to be chaotic.

Furthermore, if the integral of the spectrum is taken from $0-\sim 1982 \mathrm{~cm}^{-1}$ (the low frequency regime) and a second integral is taken from $1982-3924 \mathrm{~cm}^{-1}$ (the high frequency regime), then for FIGURE 10, the low frequency/high frequency percentages are $0 / 100$, whereas for FIGURE 11 , the ratio is $24 / 76$. Clearly, the initial excitation (which evidently was of the high frequency modes only because the low temperature spectrum shows only high frequency peaks) has remained localized in the quasi- 
periodic case and has begun to redistribute among all modes in the chaotic case. In solid polymeric materials, the low frequency modes are known as acoustical modes and the high frequency modes are known as optical modes. The technique proposed here should be useful in studying the onset of efficient energy transfer from the optical modes (perhaps excited by laser light) to acoustical modes (which can lead, upon excitation, to damage in the solids).

We note, as a final comment, that in our studies we have found that the temperature at which the chaotic limit is reached has a more complicated dependence upon the chain length than has been seen before for the atomic chains. Preliminary calculations indicate that the temperature at which the spectra become chaotic increases with increasing chain length up to a point and then essentially remains constant or drops somewhat as the chain length is further increased. Work is in progress to explore these results more fully, as well as applying these energy transfer ideas to solid polyethylene and graphite.

\section{CONCLUSIONS}

In this discussion, we have outlined the semiclassical spectral method and a wide variety of applications. Although this technique was first developed ten years ago, it has proved to be tremendously successful as a tool used in dynamics problems. Applications include problems in nonlinear dynamics, molecular and atomic spectra, surface science, astronomy and stellar dynamics, nuclear physics, and polymer physics. Innovation in the high resolution spectral method in place of the fast Fourier transform may, in the future, make possible more accurate calculations with shorter trajectories. As utilization of this technique becomes more routine, we expect many other applications to be reported in the scientific literature.

\section{ACKNOWLEDGMENTS}

We wish to acknowledge R. A. Marcus, W-K. Liu, S. K. Knudson, J. D. McDonald, J. R. Stine, and F. X. Hartman for numerous helpful discussions. We also thank Ceci Steele for her excellent secretarial help.

\section{REFERENCES}

1. Robinson, P. J. \& K. A. Holbrook. 1972. Unimolecular Reactions. Wiley. New York; Forst, W. 1973. Unimolecular Reactions. Academic Press. New York.

2. Ambartzumian, R. V. \& V.S. Letokhov. 1977. Chemical and Biochemical Applications of Lasers. Vol. 3. C. B. Moore, Ed.: 1. Academic Press. New York; Letokhov, V. S. 1977. Ann. Rev. Phys. Chem. 28: 133; Schulz, P. A., AA. S. Sudb $\emptyset$, D. J. KraJNovich, H. S. KwoK, Y. R. Shen \& Y. T. Lee. 1979. Ann. Rev. Phys. Chem. 30: 379.

3. NoID, D. W., F. X. HARTMANN \& M. L. KoszYKowSKI. 1986. In Advances in Laser Science II. W. C. Stwalley, Ed. AIP Conf. Proceedings.

4. Levy, D. H. 1980. Ann. Rev. Phys. Chem. 31: 197.

5. NolD, D. W., M. L. Koszykowski \& R. A. Marcus. 1977. J. Chem. Phys. 67: 404-408.

6. Noid, D. W., M. L. KoszykowsKi \& R. A. Marcus. 1981. Ann. Rev. Phys. Chem. 32: 267 . 
7. Einstein, A. 1917. Verh. Dtsch. Phys. Ges. 19: 82.

8. Keller, J. B. 1958. Ann. Phys. N.Y. 4: 180.

9. Koszykowski, M. L., D. W. Nold \& R. A. Marcus. 1982. J. Phys. Chem. 86: 21132117.

10. Wardlaw, D., D. W. Nold \& R. A. Marcus. 1984. J. Phys. Chem. 88: 536-547.

11. Herbst, E. \& D. W. NoID. 1984. Chem. Phys. Lett. 109: 559-562.

12. Noid, D. W. \& M. L. KoszykowSKI. 1980. Chem. Phys. Lett. 78: 114-117; NoID, D. W., S. K. KNudson \& J. B. Delos. 1983. Chem. Phys. Lett. 100: 367-370.

13. LIU, W-K., D. W. NoID \& M. L. KoszyKowsKI. 1982. In Intramolecular Dynamics. J. Jortner \& B. Pullman, Eds.: 191-203. Holland. Dordrecht.

14. STINE, J. R. \& D. W. NoID. 1983. J. Chem. Phys. 78: 1876-1883.

15. STINE, J. R. \& D. W. NoID. 1983. J. Chem. Phys. 78: 3647-3651.

16. Hansel, K. D. 1978. Chem. Phys. 33: 35; Hansel, K. D. 1979. Laser Induced Processes in Molecules. K. L. Kompa \& S. D. Smith, Eds.: 145. Springer-Verlag. Berlin/New York.

17. Sмith, A. D., W-K. Liu \& D. W. Noid. 1984. Chem. Phys. 89: 345-352.

18. Ratner, M. A. \& R. B. Gerber. 1979. Chem. Phys. Lett. 68: 195.

19. POPPE, D. 1980. Chem. Phys. 45: 371.

20. NoID, D. W. \& J. R. Stine. 1982. J. Chem. Phys. 76: 4947-4951.

21. Martin, D. L. \& R. E. Wyatt. 1982. Chem. Phys. 64: 203.

22. Powell, G. E. \& I. C. Percival. 1979. J. Phys. A12: 2053.

23. Farantos, S. C. \& J. N. Murrell. 1981. Chem. Phys. 55: 205.

24. LehmanN, K. K., G. J. Scherer \& W. Klemperer. 1982. J. Chem. Phys. 76: 6441.

25. Swamy, K. N. \& W. L. Hase. 1982. Chem. Phys. Lett. 92: 371.

26. Brickmann, J., R. Pfeiffer \& P. C. Schmidt. 1984. Ber. Bunsenges. Phys. Chem. 88: 382.

27. Lopez, V., V. Fairen, S. M. Lederman \& R. A. Marcus. 1986. J. Chem. Phys. 84: 5494.

28. Demontis, P., G. B. Suffritti, E. S. Fois \& A. Gamba. 1986. Chem. Phys. Lett. 127(N5): 456-461.

29. Koszykowski, M. L., W-K. Liu \& D. W. Noid. 1982. J. Chem. Phys. 77: 2836-2840.

30. Koszykowski, M. L., D. W. Noid, M. TABor \& R. A. Marcus. 1981. J. Chem. Phys. 74: $2530-2535$.

31. NoId, D. W., M. L. Koszykowski, M. Tabor \& R. A. Marcus. 1980. J. Chem. Phys. 72: 6169-6175.

32. NoID, D. W., M. L. Koszykowski \& R. A. Marcus. 1983. J. Chem. Phys. 78: 40184024.

33. Noid, D. W., S. K. Knudson, M. L. Koszykowski \& R. J. Renka. 1986. J. Phys. Chem. 90: 6135-6138.

34. Muckerman, J. T., D. W. Noid \& M. S. Child. 1983. J. Chem. Phys. 78: 3981-3989.

35. The latest version of the Gaussian 80 code is available from QCPE.

36. Koszykowski, M. L., P. Thiele, F. Hoffman, J. S. Binkley \& D. W. Noid. 1982. J. Am. Vac. Soc.

37. Tully, J. C., Y. J. Chabal, K. Raghavacharı, J. M. Bowman \& R. R. Lucchese. 1985. Phys. Rev. B. Condensed Matter 31: 1184.

38. Gadzuk, J. W. 1986. J. Electron Spectrosc. Relat. Phenom. 38: 233.

39. Adams, J. E. 1986. J. Chem. Phys. 84: 3589.

40. Knudson, S. K. \& D. W. NolD. 1984. Chem. Phys. 89: 353-361.

41. Davydov, A. S. 1968. Quantum Mechanics, section 94. Addison-Wesley. Reading, Massachusetts.

42. Koszykowski, M. L., D. W. Noid, F. X. Hartmann \& G. A. Pfeffer. 1987. J. Nucl. Phys. In press.

43. LANGER, R. G. 1937. Phys. Rev. 51: 669.

44. Diana, E., L. Galgani, M. Casartelli, G. Casati \& A. Scotti. 1977. Theor. Math. Phys. 29: 1022; Carotta, M. C., C. Ferrario, G. LoVecchio \& L. Galgani. 1978. Phys. Rev. A17: 786; JACKSON, E. A. 1978. Rocky Mount. J. Math. 8: 127; Galgani, L. \& G. LoVecchio. 1979. Nuovo Cimento B52: 1.

45. Henry, B. I. \& J. OitmaA. 1985. Aust. J. Phys. 38: 171; Henry, B. I. \& J. OitmaA. 1985. Aust. J. Phys. 38: 191. 